\title{
STUDIES ON THE CAUSAL ROLE OF HYPOALBUMINEMIA IN EXPERIMENTAL NEPHROTIC HYPERLIPEMIA ${ }^{1}$
}

\author{
By WALTER HEYMANN, GERALD NASH, CAROLINE GILKEY, AND \\ MILDRED LEWIS \\ (From the Department of Pediatrics, Western Reserve University, School of Medicine, \\ Cleveland, Ohio)
}

(Submitted for publication November 13, 1957; accepted February 6, 1958)

Previous work on the pathogenesis of the nephrotic hyperlipemia, in rats injected with antirat kidney sera obtained from rabbits (nephrotoxic serum, NTS), has indicated that the increase in plasma lipid concentration precedes the development of hypoproteinemia (1). They furthermore have shown that the thyroid, pituitary, adrenals, ovaries, testes and pancreas glands are not essential for its elicitation (2); that subtotal hepatectomy inhibits its development(3); and that in contradistinction to other fat depots, the hepatic lipid values are decreased (4). It was also shown that the metabolic utilization of $\mathrm{C}^{14}$ labeled trilaurin and the clearance of this substance from the blood stream after intravenous injection proceeded at normal rates (5). It was finally observed that renal tissue is essential for its elicitation, and that the severity of the hyperlipemia is related to the amount of present nephrotic kidney tissue (6).

Recently Rosenman, Friedman, and Byers have stated (7) that "the hyperlipemia observed in the experimental nephrotic syndrome is initiated and maintained by the external renal loss of plasma albumin."

The observations reported in the following studies were stimulated by the work of these authors. They are concerned with: a) relation of severity of hyperlipemia to severity of hypoalbuminemia ; b) time relationship in the development of hyperlipemia and hypoalbuminemia; and c) the effect of saline, protein-free control urine, nephrotic urine, and dextran solutions on serum cholesterol and total lipid concentrations in nephrotic rats.

\section{METHODS AND PROCEDURES}

Rats of the Sprague-Dawley strain were used. They were fed Friskies and housed in single cages. Methods used to produce the nephrotic syndrome in rats and to

\footnotetext{
1 Aided by grants of the Cleveland Area Heart Society and the Northern Ohio Chapter of the National Nephrosis Foundation.
}

determine the chemical data reported in this study have been described previously (8-12). Blood for analysis was obtained after food had been withheld for 16 hours.

A first group of studies was designed to establish a possible relationship between severity of hypoalbuminemia and hyperlipemia in the experimental nephrotic syndrome. Serum protein, serum albumin, and total lipid values were obtained in 19 nephrotic rats of our colony in whom nephrotic renal disease had persisted uninterruptedly for 1 to 11 months. These animals had been studied with weekly quantitative urinary protein determinations and monthly blood pressure readings. Their food intake was normal and their weight curves were indistinguishable from control animals.

A second group of studies was planned to investigate the time relationship in the development of hypoalbuminemia and hyperlipemia within the first 24 hours after injection of NTS. Twenty-six rats of the same weight. and sex were used for this purpose. They were given NTS by intravenous injection. The potency of the two batches of pooled sera used in these experiments had been previously established by bio-assay. In amounts of 0.69 and $1.38 \mathrm{ml}$. per $100 \mathrm{Gm}$. rat, they regularly induced nephrotic renal disease of equal severity, inducing proteinuria and hyperlipemia within the first 24 hours. The animals were killed $2,4,6,8$ and 24 hours after injection. It was essential to obtain these values from the 26 separate animals killed in the quoted intervals after the injection of NTS, because it had been noted that in rats the removal of 3 to $4 \mathrm{ml}$. of blood alone may decrease blood lipid and cholesterol values appreciably for 3 to 5 days.

Rosenman, Friedman, and Byers (7) have shown that when the loss of urinary proteins was prevented by ligation of the ureter on one side and lower vena cava-ureter anastomosis on the other side in rats injected with NTS, the development of hyperlipemia was inhibited. The intravenous infusion of bovine albumin acted similarly and diminished the degree of hyperlipemia in rats with established nephrotic renal disease. It seemed necessary to us to control these experiments by studying the effect of nonprotein-containing rat urine, and also to investigate the effect of dextran instead of albumin on the hyperlipemia of nephrotic rats. For this reason, experiments were planned to study the effect of bleeding, injections of saline, control and nephrotic urines, and dextran solutions $^{2}$ on the nephrotic hyperlipemia (Table I). Forty-

\footnotetext{
2 Plavolex $\$$, Wyeth ( 6 per cent dextran in 0.9 per cent $\mathrm{NaCl}$ solution).
} 
three nonedematous nephrotic rats were used. These had been injected with antikidney serum one and one-half to seven months prior to the first bleeding. They had uninterrupted proteinuria (100 to $400 \mathrm{mg}$. per 24 hours), ate well, gained normally in weight, and all had marked hyperlipemia with values ranging -for total lipids from 1,050 to $2,300 \mathrm{mg}$. per cent and for cholesterol from 160 to $420 \mathrm{mg}$. per cent. For reasons quoted above bleeding was spaced six to eight days apart. Initial values were obtained three days prior to the onset of treatment, and 3.5 to $4 \mathrm{ml}$. of blood was obtained from the infraorbital sinus (13) every six to eight days thereafter for three to four weeks. All solutions were given by intraperitoneal injections for 10 consecutive days in amounts of $4 \mathrm{ml}$. twice daily. Nephrotic and protein-free control urines were collected after $10 \mathrm{mg}$. of streptomycin and $10 \mathrm{mg}$. of tetracycline (Achromycin 8 ) per $20 \mathrm{ml}$. of urine had been added to the sterilized recipient jar. The samples were then filtered through a No. 6 Seitz filter and urinary cultures were obtained to assure sterility.

\section{RESULTS}

Figure 1 represents serum protein and serum albumin values (ordinate) related to the concentration of serum total lipids (abscissa) obtained in 19 nephrotic rats with well-established disease of 1 to 11 months' duration. It is evident from these results that in these animals there is no correlation between severity of hypoproteinemia or hypoalbuminemia and severity of hyperlipemia.

Figure 2 represents serum total lipid, cholesterol, serum protein and albumin values obtained in 26 rats 2, 4, 6, 8 and 24 hours after the intravenous injection of antikidney sera obtained from rabbits. From the 30 rats originally injected, 4 rats were killed accidentally and were not available for bleeding after 8 and 24 hours. It can be seen (Figure 2) that total lipid and cholesterol values were increased beyond values ever obtained in control rats in some instances two hours, in most instances four hours, and in all instances eight hours after the injection of NTS. Serum albumin concentration, however, remained within the range of normal without exception for six hours after NTS injection. After eight hours, when hyperlipemia was established in all instances, only one of four albumin values was decreased below normal range.

Table I summarizes the results obtained in 43 nonedematous nephrotic rats which were bled every six to eight days without being subjected to any intraperitoneal injections (eight rats, column

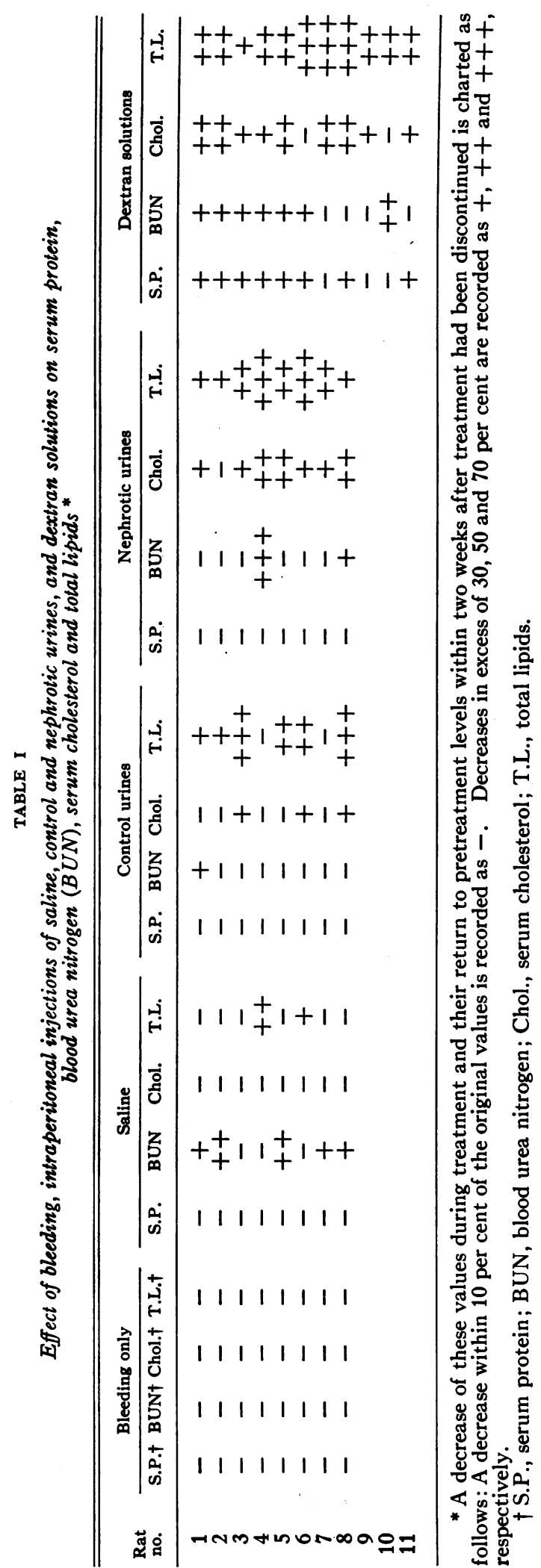


1) ; rats which were bled in identical intervals and were given $4 \mathrm{ml}$. of saline twice daily for 10 days (eight rats, column 2); rats which received $4 \mathrm{ml}$. of protein-free urine twice daily (eight rats, column 3); protein-containing nephrotic urine (eight rats, column 4); and dextran solution (11 rats, column 5) by intraperitoneal injections. If serum protein, blood urea nitrogen, cholesterol or total lipid values decreased to 30 to 50 per cent of the original value obtained three days prior to the onset of treatment, and if they returned to pretreatment levels within two weeks after treatment had been discontinued, the result is listed in Table I as + . If the decrease amounted to 50 to 70 per cent, it is registered as ++ , and in excess of 70 per cent as +++ .

It can be seen (Table I, column 1) that the removal of 3 to $4 \mathrm{ml}$. of blood every six to eight days does not alter the concentration of any of the serum constituents examined. Considering cholesterol and total lipid values, it can be noted (Table I, column 2) that total lipid values decrease temporarily only twice out of eight times during the 10 day period of saline administration, whereas the cholesterol concentration was not affected. The intraperitoneal injection of $8 \mathrm{ml}$. of protein-free control urine for 10 days decreased the elevated cholesterol values three times and the hyperlipemic total lipid values six times of eight such experiments. The magnitude of this effect was the same as when protein-containing nephrotic urine was used (Table I, column 4). The only difference was that in nephrotic rats treated with nephrotic urines, the concentration of total lipids decreased in each of the eight experiments and the cholesterol values seven out of eight times. When dextran solutions were used, the temporary decrease of lipid and cholesterol was as regularly and markedly noted as in the studies using protein-containing nephrotic urines. Because of the osmotic activity of dextran solutions, hematocrit readings were obtained at each bleeding in 3 of the 11 rats. Even though an initial decrease from 46,37 and 43 , respectively, to 30 was noted in each instance, no correlation with total lipid or cholesterol concentration was noted. While the decreased hematocrit readings did not return to normal during the period of observation, the cholesterol and total lipid values increased to the pretreatment levels within a week after the administration of dextran solutions had been discontinued. It should be re-emphasized that none of the nephrotic rats used in these studies was in the edematous phase of the disease.

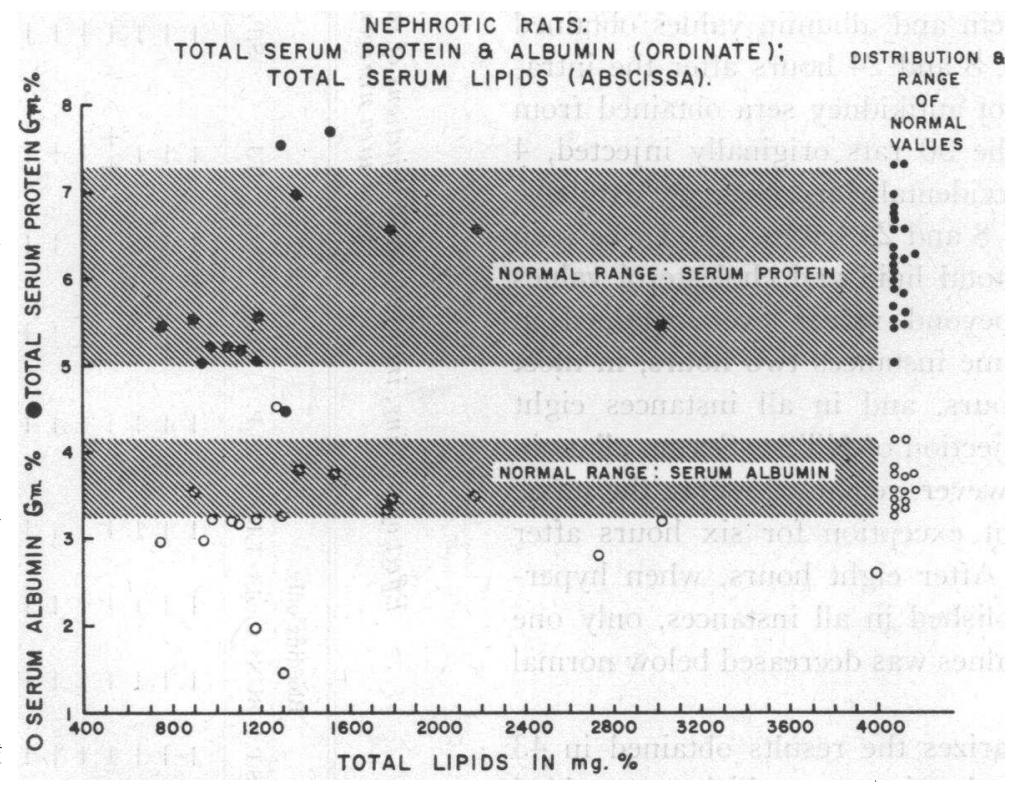

Fig. 1. Correlation of Serum Protein and Serum Albumin Concentration (Ordinate) With Total Lipid Values (Abscissa) in Sera of 19 Nephrotic Rats 


\section{DISCUSSION}

If hypoalbuminemia were the only cause for the nephrotic hyperlipemia, an inverse relationship between severity of plasma albumin depletion and severity of the hyperlipemia could have been expected. That this has not been established (Figure 1) does not, however, eliminate the hypoalbuminemia as one of several factors that may pathogenetically be at play in the elicitation of the nephrotic hyperlipemia.

It has furthermore been shown (Figure 2) that hypoalbuminemia does not precede the development of hyperlipemia if studies are carried out within the first two to four hours after the injection of NTS. It can, in fact, be seen (Figure 2) that hypoalbuminemia succeeds the development of the hyperlipemia in most instances. If hyperlipemia can be noted within two hours after the administration of NTS it is almost inconceivable that hypoalbuminemia could have developed prior to that period of time.

The studies summarized in Table I show that the concentration of serum total lipids and cholesterol can be rather easily decreased by various procedures. The withdrawal of blood alone has been mentioned before. If spaced six days apart, we feel reassured (Table I, column 1) that this does not play a role in our studies. The injection of saline alone (Table I, column 2) can, however, occasionally decrease the total lipid concentration without affecting cholesterol values. Protein-free urine (column 3 ) decreased total lipid concentration markedly in six of eight experiments. In these six instances it would be difficult to distinguish the results from those reproduced in column 4 (Table I) where protein-containing nephrotic urine was used. It is thus probable that in the studies of Rosenman, Friedman, and Byers (7) similar results, possibly differing in intensity only in some of their experiments, would have been obtained if the nephrotic rats would have been infused with urine obtained from healthy control animals instead of having been subjected to a vena cava-ureteral anastomosis. It also is evident from column 5 (Table I) that dextran solution would have had the same effect on blood lipid and cholesterol concentration as that observed by the authors (7) when they infused nephrotic rats with albumin solution. We may assume that this

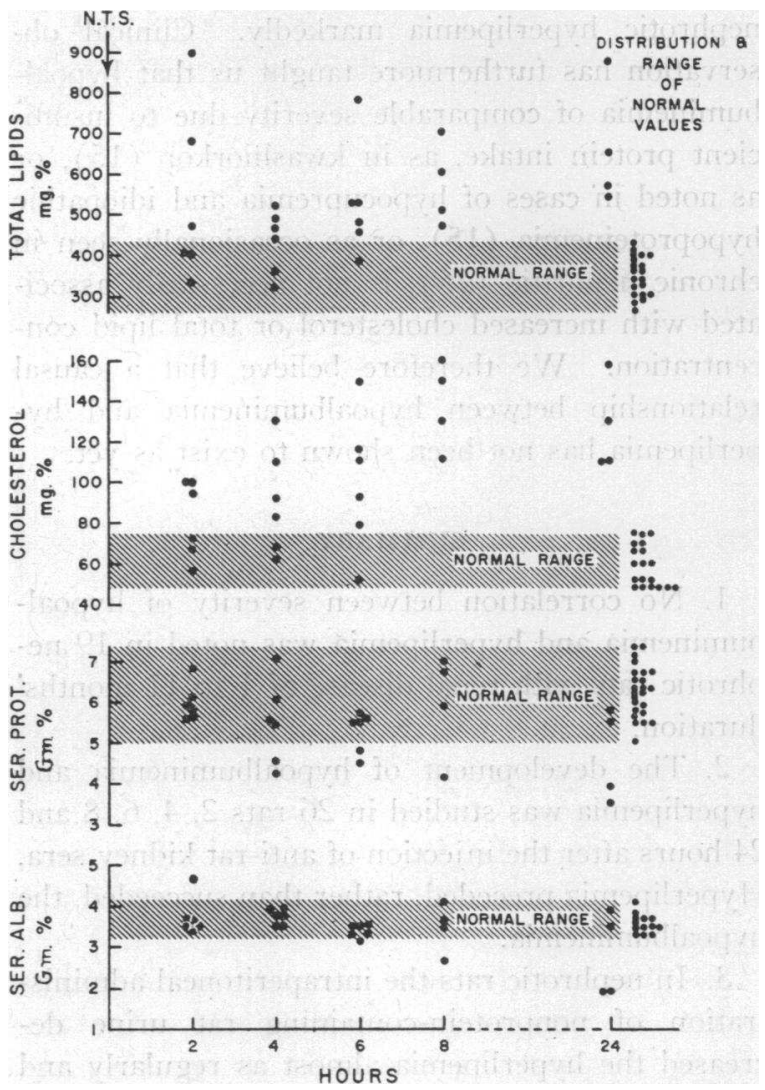

Fig. 2. Total Lipid, Cholesterol, Serum Protein and Serum Albumin Values Obtained in 26 Rats 2, 4, 6, 8 AND 24 Hours, Respectively, After the INTRAVENOUS INJECTION OF NePhrotoxic SERUM (NTS)

The shaded areas indicate the range of values obtained in 12 to 21 healthy rats of the same age and weight.

effect of dextran solutions is not due to hemodilution in that only nonedematous animals were used and hematocrit readings were unrelated to the observed lipid and cholesterol values obtained. The mechanisms of the hyperlipemia-reducing action of dextran is unclear. It has also been described by Cohen and Tudhope (14) in non-nephrotic human patients with hypercholesterolemia.

The coexistence of hypoalbuminemia and hyperlipemia is well-established in all forms of the nephrotic syndrome. We believe, however, that a causal relationship between them has not been demonstrated, in that: a) There is no relationship between severity of hypoalbuminemia and hyperlipemia ; b) hyperlipemia does not succeed, but usually precedes the decrease in serum albumin concentration; and c) protein-free urine and dextran instead of albumin solutions decrease the 
nephrotic hyperlipemia markedly. Clinical observation has furthermore taught us that hypoalbuminemia of comparable severity due to insufficient protein intake, as in kwashiorkor (15), or as noted in cases of hypocupremia and idiopathic hypoproteinemia (15), or as occasionally seen in chronic nephritis (16), is not necessarily associated with increased cholesterol or total lipid concentration. We therefore believe that a causal relationship between hypoalbuminemia and hyperlipemia has not been shown to exist as yet.

\section{SUMMARY}

1. No correlation between severity of hypoalbuminemia and hyperlipemia was noted in 19 nephrotic rats with renal disease of 1 to 11 months' duration.

2. The development of hypoalbuminemia and hyperlipemia was studied in 26 rats $2,4,6,8$ and 24 hours after the injection of anti-rat kidney sera. Hyperlipemia preceded, rather than succeeded, the hypoalbuminemia.

3. In nephrotic rats the intraperitoneal administration of nonprotein-containing rat urine decreased the hyperlipemia almost as regularly and markedly as when protein-containing nephrotic urines were used. The effect of dextran solutions on the experimental hyperlipemia was marked and regularly noted.

4. It is concluded that a causal relationship between hypoalbuminemia and hyperlipemia in the experimental renal disease of rats has as yet not been established.

\section{REFERENCES}

1. Heymann, W., and Hackel, D. B. The early development of anatomic and blood chemistry changes in the nephrotic syndrome in rats. J. Lab. clin. Med. 1952, 39, 429.
2. Heymann, W., Hackel, D. B., Gilkey, C., and Salehar, M. Relation of pituitary and adrenal glands to the nephrotic syndrome in rats. Lab. Invest. 1953, 2, 423.

3. Heymann, W., and Hackel, D. B. Role of liver in pathogenesis of experimental nephrotic hyperlipemia. Metabolism 1955, 4, 258.

4. Heymann, W., and Hackel, D. B. Hepatic and extrahepatic depot lipids in rats with experimental nephrotic hyperlipemia. Metabolism 1957, 6, 169.

5. Heymann, W., Matthews, L. W., Lemm, J., Olynyk, P., Salehar, M., and Gilkey, C. Fat metabolism in nephrotic hyperlipemia. Metabolism 1954, 3, 27.

6. Heymann, W., and Hackel, D. B. Role of kidney in pathogenesis of experimental nephrotic hyperlipemia in rats. Proc. Soc. exp. Biol. (N. Y.) 1955, 89, 329.

7. Rosenman, R. H., Friedman, M., and Byers, S. O. The causal role of plasma albumin deficiency in experimental nephrotic hyperlipemia and hypercholesteremia. J. clin. Invest. 1956, 35, 522.

8. Heymann, W., and Lund, H. Z. Nephrotic syndrome in rats. Pediatrics 1951, 7, 691.

9. Weichselbaum, T. E. Accurate and rapid method for determination of proteins in small amounts of blood serum and plasma. Amer. J. clin. Path. Tech. Sect. 1946, 10, 40.

10. Wilson, W. R., and Hanner, J. P. Changes of total lipid and iodine number of blood fat in alimentary lipemia. J. biol. Chem. 1934, 106, 323.

11. Schoenheimer, R., and Sperry, W. M. A micromethod for the determination of free and combined cholesterol. J. biol. Chem. 1934, 106, 745.

12. Archibald, R. M. Colorimetric determination of urea. J. biol. Chem. 1945, 157, 507.

13. Stone, S. H. Method for obtaining venous blood from the orbital sinus of the rat or mouse. Science 1954, 119, 100.

14. Cohen, H., and Tudhope, G. R. Dextran sulphate: Use as an anticoagulant, and action in lowering serum cholesterol. Brit. med. J. 1956, 2, 1023.

15. Cooke, R., Goodman, H., and Rapoport, M. Discussion in Proceedings of the Eighth Annual Conference on the Nephrotic Syndrome, J. Metcoff, Ed. The National Nephrosis Foundation, Inc., 1956, p. 98.

16. Heymann, W. Unpublished data. 